\title{
Simulation teaching method in Engineering Optics
}

\section{Qieni Lu, Yi Wang, Hongbin Li}

Qieni Lu, Yi Wang M.D., Hongbin Li Sr., "Simulation teaching method in Engineering Optics," Proc. SPIE 10452, 14th Conference on Education and Training in Optics and Photonics: ETOP 2017, 104526J (16 August 2017); doi: $10.1117 / 12.2269959$

SDIE Event: 14th Conference on Education and Training in Optics and Photonics, ETOP 2017, 2017, Hangzhou, China 


\title{
Simulation Teaching Method in "Engineering Optics"
}

\author{
Lu Qieni ${ }^{1}$, Wang Yi ${ }^{1}$, Li Hongbin ${ }^{2}$ \\ ${ }^{1}$ School of Precision Instruments and Optoelectronics Engineering, Tianjin University \\ Key Laboratory of Opto-electronics Information Technology, \\ Ministry of Education, Tianjin 300072, China \\ ${ }^{2}$ School of Foreign Languages and Literature, Tianjin University
}

\begin{abstract}
We here introduce a pedagogical method of theoretical simulation as one major means of the teaching process of "Engineering Optics" in course quality improvement action plan $(\mathrm{Qc})$ in our school. Students, in groups of three to five, complete simulations of interference, diffraction, electromagnetism and polarization of light; each student is evaluated and scored in light of his performance in the interviews between the teacher and the student, and each student can opt to be interviewed many times until he is satisfied with his score and learning. After three years of Qc practice, the remarkable teaching and learning effect is obatined. Such theoretical simulation experiment is a very valuable teaching method worthwhile for physical optics which is highly theoretical and abstruse. This teaching methodology works well in training students as to how to ask questions and how to solve problems, which can also stimulate their interest in research learning and their initiative to develop their self-confidence and sense of innovation.
\end{abstract}

Keywords: teaching method, teaching practice, theoretical simulation teaching method, score assessment

\section{INTRODUCTION}

"Engineering Optics" is major fundamental specialized course for programs like optoelectronic information science and engineering, instrumentation and other similar programs of institutes of higher education in China. The course of physical (wave) optics mainly instructs fundamental theories and methods of physical optics and its application technology, the main contents of which are fundamental laws of electromagnetic theory, interference and interference system, diffraction and polarization of light. This course enables students to command basic concepts, laws and typical systems of optics, and also cultivate their ability to ask questions and solve optical problems and their innovation sense and then they can work and research in the field of optical-electric information processing, optical communications and optical measurement.

Tianjin University "Engineering Optics" is a national-level excellent course project, a major required course for juniors in our school. As of 2004, a series of teching reforms have been undertaken and many innovative teching methods are practised with excellent teching results, and a number of teching reform projects have been completed [1-3]. The course quality improvement action plan (Qc) was lauched in 2013 years, and excellent teching results have been obatined over the three years. In this paper, we mainly introduce the key teaching procedure the theoretical simulation (a comprehensive educating and training teaching method) and its achievements as the Qc practice goes on.

\section{A TEACHING METHOD OF THEORETICAL SIMULATION}

Theoretical simulation method is very important and increasingly widely adopted in scientific research $[4,5]$. By simulating physical processes and phenomena, researchers can determine the optimal parameters, design the optimal optical system, evaluate the performance of the algorithm and model and so on for further physical implementation. For example, in particle size measurement where interferometric particle imaging is used, the performance of the extracted fringe frequency algorithm is evaluated by simulated interferogram of a particle, and then the particle diameter can be subsequently measured. This simulation method has also been infiltrated to teaching [6-8]. In physical optics instruction, theoretical simulation is to move the optical experiment to computer, optical experiments which students previously need to practice in the lab can now be carried out by computer and optical phenomena, such as Young's interference, can be observed by simulation, then students can find that the fringe spacing depends on wavelength, the separation of the slits, and the distance between the slits and the observation plane (see Fig.3). This manner of theoretical simulation is

14th Conference on Education and Training in Optics and Photonics: ETOP 2017, edited by Xu Liu,

Xi-Cheng Zhang, Proc. of SPIE Vol. 10452, 104526J - (c) 2017 ICO, IEEE, OSA, SPIE

CCC code: $0277-786 \mathrm{X} / 17 / \$ 18 \cdot$ doi: $10.1117 / 12.2269959$ 
analogous in the sense that students do the optical experiment actually in lab. Optical concepts and laws abstruse and difficult to understand are described intuitively and vividly by simulation experiment, which promotes their understanding and also predicts the experimental results in advance. In addition, the combination of traditional optical teaching and computer simulation makes the optical measurement and analysis quantitative, which is also a current trend of optical experiment teaching development. For physical optics a highly theoretical course, theoretical simulation plays an important role in physical optics teaching, and is a comprehensive training and education of student' learning and research ability.

Simulation topics and subjects are prepared by teachers, which covers widely across the course, such as, double-beam interference, which are general scientific topics for students to explore their wide different applications in reality. For such important fundmental difficult course, students form study groups of three or five members to complete simulation experiments. The resluts are submitted by groups.

\section{EXAMPLES}

In this section, we display some examples of numerical simulation. The light field distribution of Fresnel diffraction and Fraunhofer diffraction are, respectively [9-11],

$$
\begin{aligned}
& u(x, y)=\frac{\exp i k z}{i \lambda z} \iint u_{0}\left(x_{0}, y_{0}\right) \exp i \frac{k}{2 z}\left[\left(x-x_{0}\right)^{2}+\left(y-y_{0}\right)^{2}\right] d x_{0} d y_{0} \\
& u(x, y)=\frac{\exp i k z}{i \lambda z} \exp i k \frac{x^{2}+y^{2}}{2 z} \iint u_{0}\left(x_{0}, y_{0}\right) \exp \left[-i \frac{k}{2 z}\left(x x_{0}+y y_{0}\right)\right] d x_{0} d y_{0}
\end{aligned}
$$

where $k=2 \pi / \lambda, \lambda$ is the wavelength of the incident light, $\left(x_{0}, y_{0}\right)$ is the plane of the diffraction screen, $(x, y)$ is the plane of observation, $\mathrm{z}$ is the distance between the plane of the diffraction screen and the observation plane, $u_{0}\left(x_{0}, y_{0}\right)$ is diffraction screen function. Fig. 1 shows Fresnel diffraction field distribution of a circular aperture at different distance $\mathrm{z}$ using Eq.(1) and MATLAB. As illustrated in Fig.1, the Fresnel diffraction pattern of the circular aperture changes and the intensity of the center point of the diffraction field distribution changes alternately with $\mathrm{z}$ increasing. As $\mathrm{z}$ increase to Fraunhofer diffraction zone, the diffraction pattern shape does not change, but only the scale change. This simulation experiment enables students to better understand Fresnel diffraction and Fraunhofer diffraction process, and that the Fresnel diffraction zone includes Fraunhofer diffraction zone, that Fraunhofer diffraction is a further approximation of Fresnel diffraction. Fig.2 gives Fraunhofer diffraction patterns of a double circular aperture obtained by using Eq.(2) and MATLAB. The top row is double circular aperture geometry and the bottom row is the diffraction patterns.
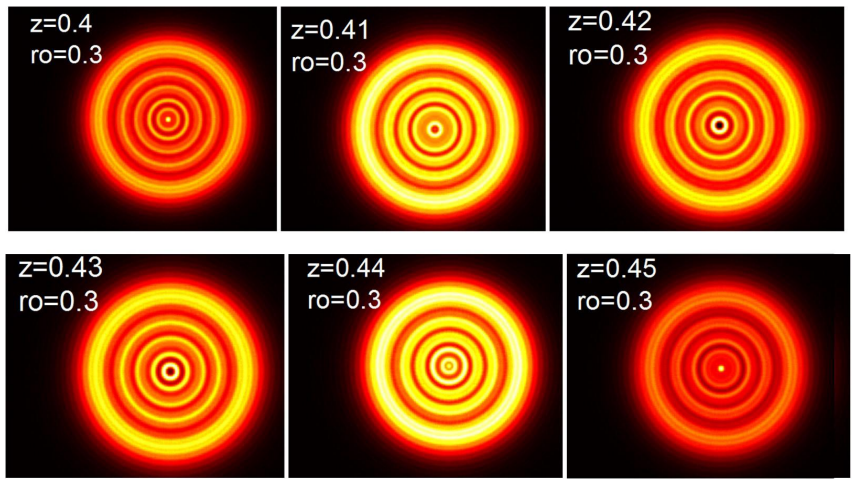

Fig.1 Fresnel diffraction patterns for a circular aperture.
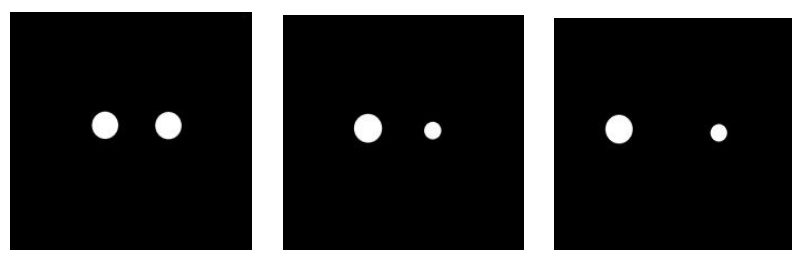

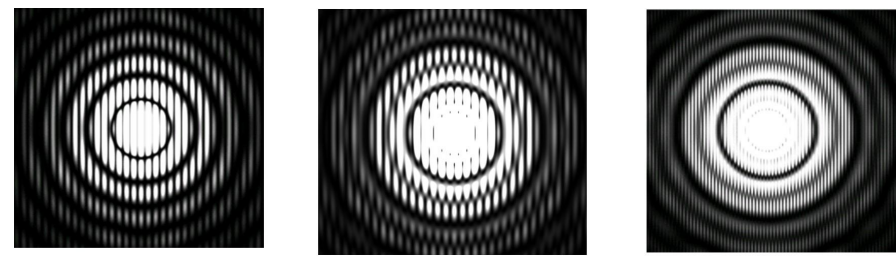

Fig.2 Double aperture geometry (top row) and Fraunhofer diffraction patterns (bottom row).

Fig. 3(a)-(c) show the two plane wave interferograms, that is grating, the fringe period of grating is $d=\lambda / 2 \sin \theta$, and frequency of grating is $f=1 / d=2 \sin \theta / \lambda$, where $\lambda$ is the wavelength, $2 \theta$ is the intersection angle between two plane waves. In Fig. 3(a), $\lambda=532 \mathrm{~nm}, 2 \theta=60^{\circ}$, Fig. $3(\mathrm{~b}), \lambda=532 \mathrm{~nm}, 2 \theta=90^{\circ}$, and Fig. $3(\mathrm{c}), \lambda=632.8 \mathrm{~nm}, 2 \theta=90^{\circ}$. For a given $\lambda$, the bigger fringe spacing with the smaller $\theta$, see Fig.3(a), and for a given $\theta$, the bigger fringe spacing with the bigger $\lambda$, see Fig.3(c). By selecting $\theta$ or/and $\lambda$, grating spacing can be changed. Fig. 3(d)-(f) show the intensity distribution, 2D and 1D Fourier spectrum of Fig.3(a), respectively. The spacing between the zero-order spectrum and first-order spectrum of the frequency spectrum is the frequency of the interference fringe, the estimated frequency $f=2.67 \mu \mathrm{m}$.

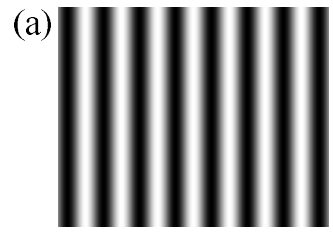

(d)

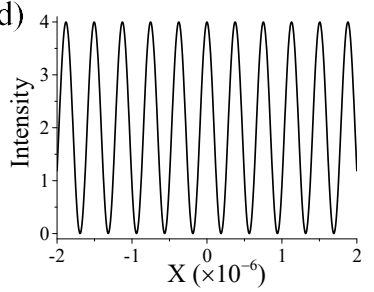

(b)

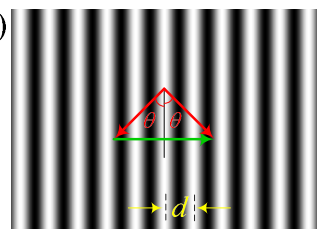

(e)

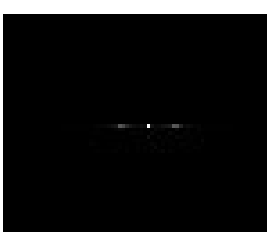

(c)
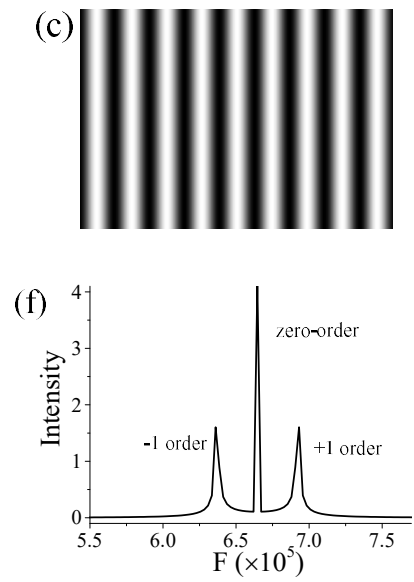

Fig.3 (a)-(c) Interferograms with different parameters; (d) the intensity distribution, (e) 2D and (f)1D frequency spectrum of (b).

\section{RESULTS EVALUATION}

The teaching method reform will be accompanied by the assessment method reform. The reform of teaching testing method is another important teaching process in Qc practice and also one of the main means revealing the effect of teaching reform. We present a method of scoring assessment and examination of a process assessment, which is different from the result testing method. The teacher assigns topics to students; student groups complete simulation and then submit it to the teacher. The teacher assesses their simulation and gives score and feedback to student groups; student groups redo it according to the reviews by the teacher and then resubmit, successively repeated until student groups are satisfied with their study and score. Each simulation topic can be redone many times and a final score of the simulation experiment is given to student groups for their simultation of the best performance; here the assessment standard by which to judge their work is the depth and breadth of their simulation experiments. After the group score is given, each individual student is evaluated and scored in light of his personal performance in the interviews between the teacher and him, and each student can opt to be interviewed many times until the student is satisfied with the score. Fig. 4 shows the assessment flowchart. It is noticed that the score of each student in groups does not exceed the the score of groups (the score of the simulation experiment). The interview contents cover the entire contents of the curriculum, including the simulation. Fig.5 shows the changes before and after Qc practice. You can see from Fig.5 that the stuedent score impoves highly by using theoretical simulation teaching manner, the $15 \%$ grade increase rate has been obatined by Qc practice, and the excellent rate has reached $42.42 \%$ in 2014 while $8.93 \%$ before ( $85-100$ are excellent) in our school. 


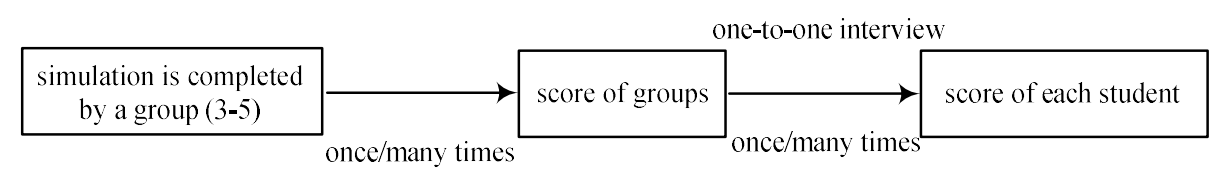

Fig. 4 Flowchart of score assessment.
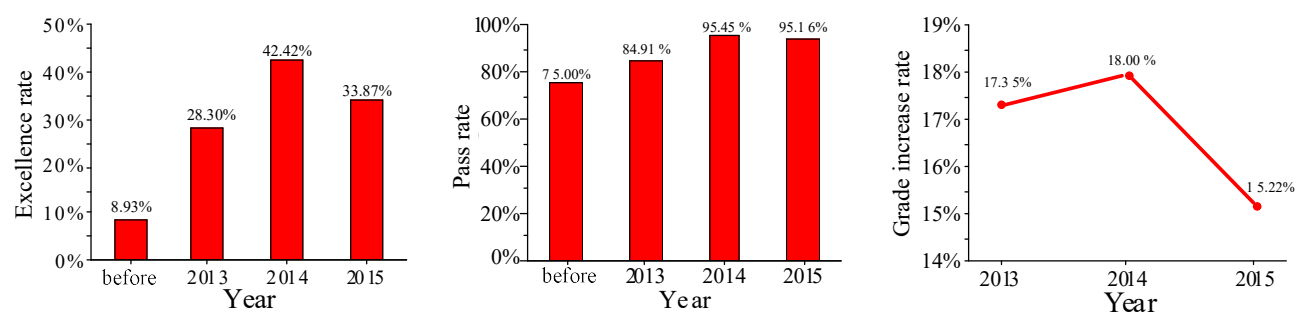

Fig.5 Results before and after using theoretical simulation teaching manner.

\section{CONCLUSION}

For physical optics being a highly theoretical course, theoretical simulation is an effective teaching method. On the one hand, by simulation, the abstract concept and the mathematical formula are converted to graphs and images, which will be helpful for student to better understand and learn the knowledge of course, and thus improve the teaching effect. On the other hand, through theoretical simulation, the experimental results are prejudged in advance. This teaching methodology also work well in training student as to how to ask questions and how to solve problems, which can stimulate students' interest in learning and initiative to develop their self-confidence and consciousness of innovation. Such theoretical simulation manner is a very valuable teaching method worthwhile adopting for physical optics which is highly theoretical and abstruse.

\section{REFERENCES}

[1] Cai Huaiyu, Yu Daoyin, and Li Qing, "The construction and reform of the excellent course of "Engineering optics"," Hi. Edu. Sci., 66, 38-40(2006).

[2] Cai Huaiyu, Yu Daoyin, Ge Baozhen, and Li Qing, "The practice and experience of completing the quality project in college of PIOE, Tianjin University,” Opt. Tech.,(Sup.) 36,124-126(2010).

[3] Lu Qieni, Cui Yuming, Cai Huiyu, and Wang Qiang, "Constructing a teaching platform of "engineering optical experiment"," Opt. Tech., (Sup.) 36,105-107(2010).

[4] Q. Lu, W. Jing, T. Lu, X. Wang, and Y. Zhang, "High-accuracy particle sizing by interferometric particle imaging," Opt. Commun. 312(2), 312-318 (2014).

[5] H. Shen, S. Coëtmellec, G. Gréhan, and M. Brunel, "Interferometric laser imaging for droplet sizing revisited: elaboration of transfer matrix models for the description of complete systems," Appl. Opt. 51(22), 5357-5368 (2012).

[6] Qu Weijuan, Optical experiment simulation with Matlab, M. S. Dissertation of Northwestern polytechnical University, 2004.

[7] Zhou Yahui, Zhu Hao, Zhou Aiping, and Deng Yan, "The design of a remote optics experiment system based on LabVIEW,” Exp. Tech. Management 23(10), 63-66(2006).

[8] Yang Huajun, Liu Jignxian, Huang Keqin, Wu Zhenhai, and Gao Hao, "Applying computer simulation in the teaching of Optics," Exp. Sci. Tech., 5(3), 101-104(2007).

[9] Yu Daoyin, Tan Henying, Engineering Optics (in Chinese), China machine press, 2006.

[10] Eugene Hecht, Cunlin Zhang adapt. Optics, 4th edition, Higher education press (Beijing China), 2005.

[11] Quantin Liang. Physical Optics (in Chinese), China machine press, 2009. 